\title{
Gastrointestinal Bleeding as a Complication in Continuous Flow Ventricular Assist Devices: A Systematic Review With Meta-Analysis
}

\author{
Edinen Asuka ${ }^{\mathrm{a}, \mathrm{d}}$, Stella Pak ${ }^{\mathrm{b}}$, Armond-Kristopher Thiess ${ }^{\mathrm{c}}$, Anthony Torres, III ${ }^{\mathrm{c}}$
}

\begin{abstract}
Background: The use of ventricular assist devices (VADs) has become predominant in this era of medicine. It is commonly used as a bridge to transplant, recovery and as a destination therapy for patients with severe heart failure, who are not responsive to maximum optimal management or ineligible for transplant. However, several complications are known to occur with the use of these devices. In this research, we will compare gastrointestinal bleeding in patients who used centrifugal flow versus axial flow VADs. We hope that the result of this meta-analysis and the review presented provide adequate information to future researchers, physicians and other healthcare professionals who are interested in this topic.
\end{abstract}

Methods: Published articles evaluated for inclusion were obtained from MEDLINE (PubMed), Cochrane, EBSCO, clinicaltrials.gov, and international clinical trials registry. This research was conducted according to the Preferred Reporting Items for Systematic Reviews and Meta-Analyses (PRISMA) guidelines. Procured articles were reviewed by two independent reviewers. Only randomized control trials and observational studies were used. Quality assessment was done with Cochrane Collaboration's tool (RoB.2 with visualization through robviz) and NewcastleOttawa Scale (NOS). Data analysis was carried out with the use of R data analysis tool (version 4.0.0; release date: April 24th, 2020).

Results: At the end of this meta-analysis, the occurrence of gastrointestinal bleeding was not significantly different between both groups; with odds ratio (OR): $0.81 ; 95 \%$ confidence interval (CI): $0.65-1.00$; $\mathrm{P}$ value $=0.05$. Between-study variance $($ Tau-squared) was zero $(0)$, standard error $(\mathrm{SE})=0.06$. The degree of heterogeneity measured with I-squared statistic was $0 \%$ (minimal). Egger's regression test

Manuscript submitted June 16, 2020, accepted June 26, 2020

Published online August 15, 2020

aAll Saints University School Of Medicine, Hillsborough St, Roseau, Dominica

${ }^{b}$ Department of Medicine, Orange Regional Medical Center, 707 East Main Street, Middletown, NY 10940, USA

${ }^{\mathrm{c}}$ Department of Medicine, Universidad Autonoma de Guadalajara, Av. Patria 1201, Lomas del Valle 45129, Zapopan, Mexico

${ }^{\mathrm{d} C o r r e s p o n d i n g ~ A u t h o r: ~ E d i n e n ~ A s u k a, ~ A l l ~ S a i n t s ~ U n i v e r s i t y ~ S c h o o l ~ O f ~ M e d i-~}$ cine, Hillsborough St, Roseau, Dominica. Email: edinenasuka@gmail.com

doi: https://doi.org/10.14740/jocmr4262 was not statistically significant, $P=0.93$. Symmetry of distribution was observed on the funnel plot. Trim and fill analysis showed no missing studies on the left; $\mathrm{SE}=1.68$.

Conclusions: The result obtained from this research indicates that the occurrence of gastrointestinal bleeding is not significantly different in both groups of patients, irrespective of the type of continuous flow VAD used. Although, the study sample used in this meta-analysis was limited.

Keywords: VAD; Continuous flow VADs; Axial flow VADs; Centrifugal flow VAD; Gastrointestinal bleeding; Biventricular Assist device; Extracorporeal Membrane Oxygenation

\section{Introduction}

Congestive heart failure (CHF) is the most common diagnosis in nearly 875,000 hospitalizations, with the most common diagnosis in hospital patients aged 65 years and older. More than half of those who develop CHF die within 5 years of diagnosis. Nearly 5 million Americans are currently living with CHF and about 26 million worldwide. CHF affects people of all ages, from children and young adults to the middle-aged and the elderly; although, it is more predominant in older age groups [1, 2]. According to the Center for Disease Control and Prevention, it was a contributing cause of one in eight deaths in the USA in 2017. The yearly economic repercussion in 2012 was reported to be $\$ 30.7$ billion including medical costs [2]. Heart transplantation is the best treatment option for these patients, especially in those with New York Heart Association (NYHA) class III - IV heart failure. Close to 100,000 individuals are in need of a heart transplant yearly but only about 2,000 heart transplants are done yearly in the USA [3]. Patients with advanced heart failure are also known to have a life expectancy of less than 2 years without mechanical circulatory support [4]. Current data show that the use of ventricular assist devices (VADs) for patient care seem to be an enormous headway in medicine, especially in regions with limited availability of organs for transplant and for high-risk patients who are ineligible for transplant. This concept which is still novel in some parts of the world can be used in a myriad of cases; some of which include management of patients awaiting heart transplantation as a form of bridge therapy. This has helped to circumvent the 


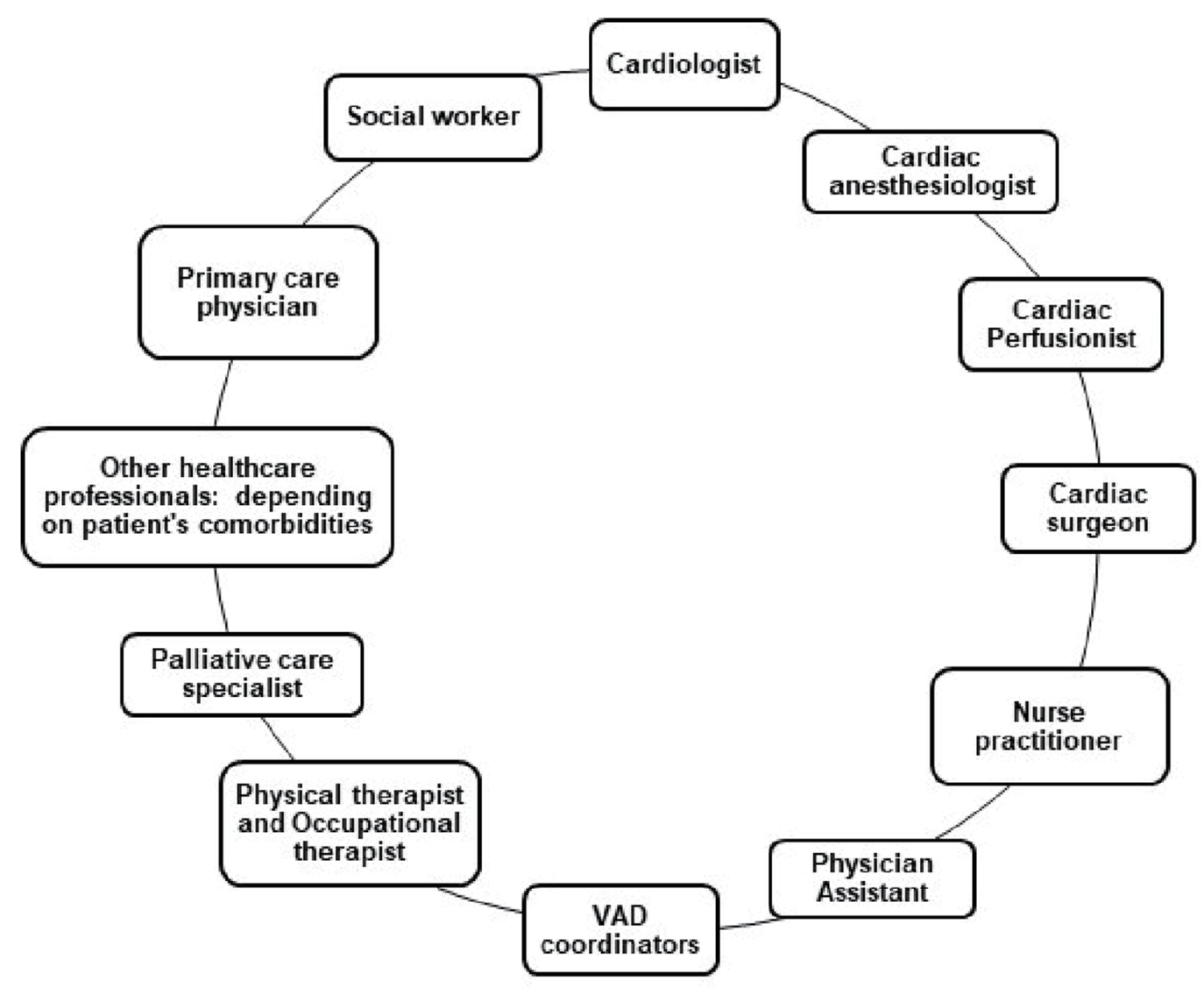

Figure 1. Multidisciplinary team commonly involved in the care of patients with VADs. VAD: ventricular assist devices.

limitation of organ availability [5-9]. It can also be used as destination therapy in dire cases of severe ventricular hypokinesis involving one or both ventricles or in patients who are poor candidates for heart transplant. In addition, VADs may be used as a short term recovery therapy for patients who have recently undergone cardiac surgery (postcardiotomy shock) and in patients with severe cardiac decompensation secondary to acute coronary syndrome or severe pulmonary hypertension. It is also used to wean patients off extracorporeal membrane oxygenation (ECMO), in refractory cases [6-10]. Despite its promising outcomes, it is associated with several complications such as infection, bleeding, neurologic events, thromboembolism, cardiac arrhythmia, psychiatric disorder, device thrombosis, pericarditis, right heart failure and renal failure The care of patients with VADs involves a multidisciplinary approach with shared decision making as illustrated in Figure 1. Prognostic scoring systems such as Lietz-Miller score and ADHERE CART model can be used for preoperative risk stratification, survival analysis and in-hospital mortality assessment [11, 12]. Medical management in these patients is maintained with anticoagulation with or without antiplatelet therapy, optimal blood pressure control and treatment of underlying comorbidities. Devices such as the HeartMate XVE do not require long-term anticoagulation with warfarin except aspirin therapy due to its fibrin-derived bioprosthetic surface [10-13]. This can be beneficial for patients with contraindications to anticoagulant therapy. Nevertheless, due to its cumbersome nature and lesser durability as compared to contemporary VAD versions, it is less commonly preferred. Blood pressure measurement is often done by Doppler ultrasonography and intra-arterial approach in both outpatient and inpatient settings respectively [14].

\section{Classifications}

There are various classifications of VADs. Herein, we will explore its numerous forms. The prosthetic ventricle of VADs can be placed inside the body (intracorporeal) or outside (paracorporeal or extracorporeal). Flow through the pump can be continuous or pulsatile $[6,7,15]$. VADs with continuous flow pumps use hydrodynamic or magnetic mechanisms to suspend and anchor their rotors while most pulsatile pumps utilize pneumatic mechanisms [15]. There are currently two types of continuous flow VADs, axial and centrifugal flow VADs. Common concepts of VADs notably available are left VAD, right VAD and biventricular assist device $[16,17]$. Although, LVADs seem to be the most commonly used, VADs can be further classified based on their mode of implantation, which can occur percutaneously or through open heart surgery (Fig. 2). Unlike ECMO, which can be used for short-term ventilatory and circulatory support; VADs are used solely for circulatory support. However, VADs require lower heparin dosing, lesser priming volume and provide better ventricular compensation 


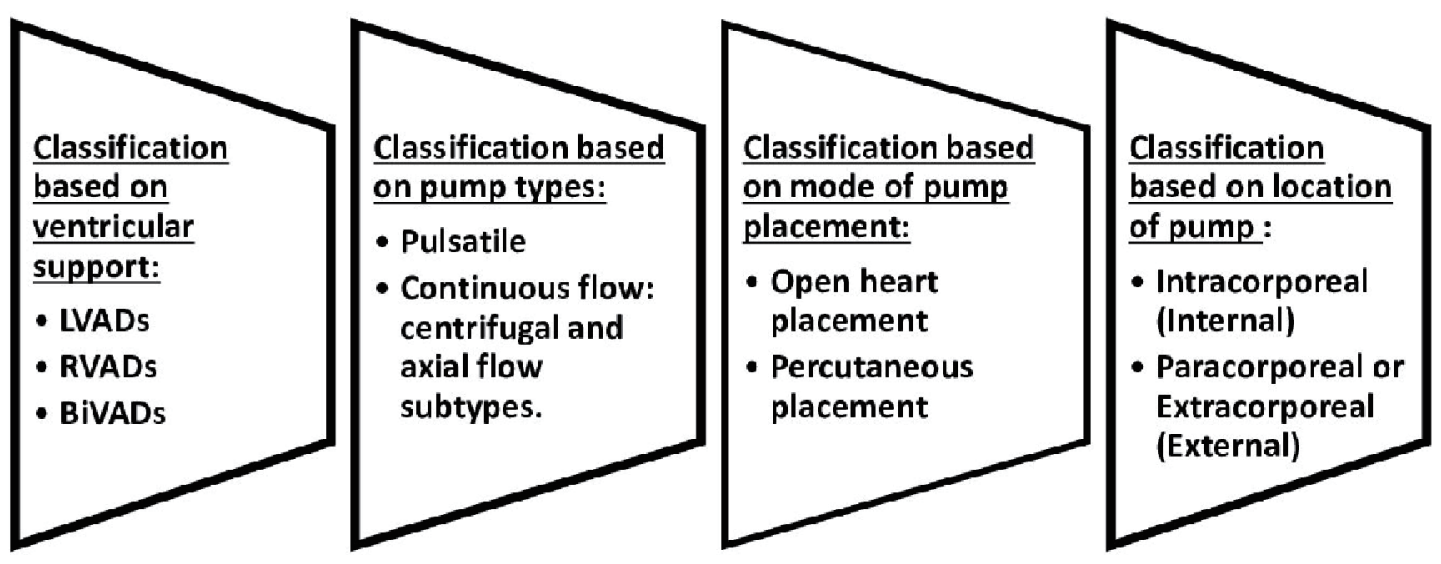

Figure 2. Classifications of ventricular assist devices.

[18]. Most VADs are portable and easily conveyed. Nevertheless, some VADs such as Berlin EXCOR are not quite portable. It is also important to note that some modernized versions of ECMOs can easily be conveyed around as well. They are currently different versions of VADs. A few to be mentioned include HeartMate 3, HeartMate II, HeartMate XVE, Jarvik, HeartWare, Thoratec Paracorporeal VAD and Thoratec Implantable VAD, Tandem heart, CentriMag, and Impella 2.5 [1518]. The conventional flow pumps are mainly used in adults because of their relative size which makes it difficult for them to be used in children and infants. Notwithstanding, there are some VADs such as Berlin Heart EXCOR designed for right and left ventricular support, which can be used in neonates, infants, children and adolescents [15-26]. For short-term circulatory support in pediatric patients, ECMO is still used as the mainstay in most health care institutions [19-26]. Other VADs that can be used in the pediatric population include Jarvik 2000 (Jarvik Heart), PediaFlow, miniature MVAD HeartWare, and CircuLite. Known alternatives to VAD therapy include heart transplant, ECMO, intra-aortic balloon pump (IABP) and total artificial heart (TAH). Most approved TAHs such as Cardiowest and Abiomed TAHs are used for long-term mechanical circulatory support. Currently, IABP and ECMO are restricted for use in short-term circulatory support [11, 12, 15, 20, 27$]$.

\section{Mechanism of action}

Early forms of VADs used mainly pneumatic or electrical sources of energy to enhance systemic propulsion of blood $[15,16,20,27]$. They were mostly pulsatile flow VADs (first generation). Subsequently, the use of continuous flow VADs were employed, starting with the axial flow VADs (second generation) before the centrifugal flow VADs (third generation). Earlier pulsatile VADs were cumbersome and less durable as compared to its modern counterparts. A complete circuitry of a VAD consists of an external set of direct current (DC) batteries or an alternating current (AC) input and a power module, system controller, driveline, pump unit, inflow and outflow cannulas (Figs. 3, 4). The system controller serves as the central control station. It is used to control the flow, speed, power and pulsatility of the pumps $[6,7,15,20$, 27]. There are several variations to the origin of the inflow and outflow cannulas. Most paracorporeal VADs have their inflow cannula placed in the left ventricle and the outflow cannula placed in the ascending aorta. For right ventricular

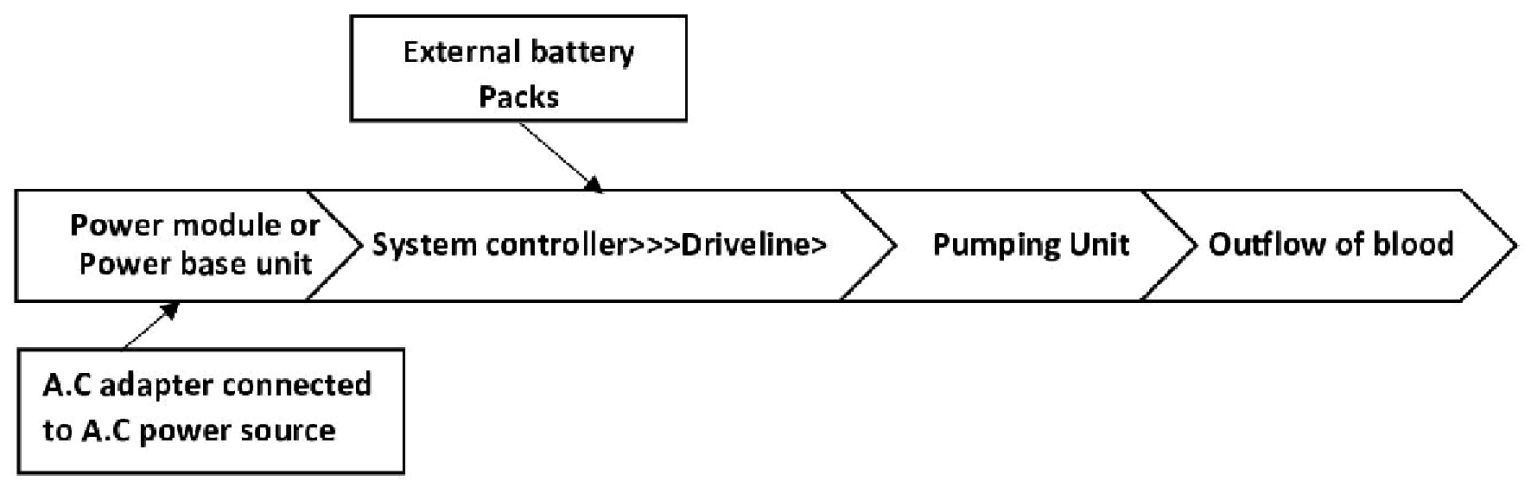

Figure 3. Schematic representation of ventricular assist device work flow using tethered (AC power source) and untethered connection (DC power source). AC: direct current; $A C$ : alternating current. 


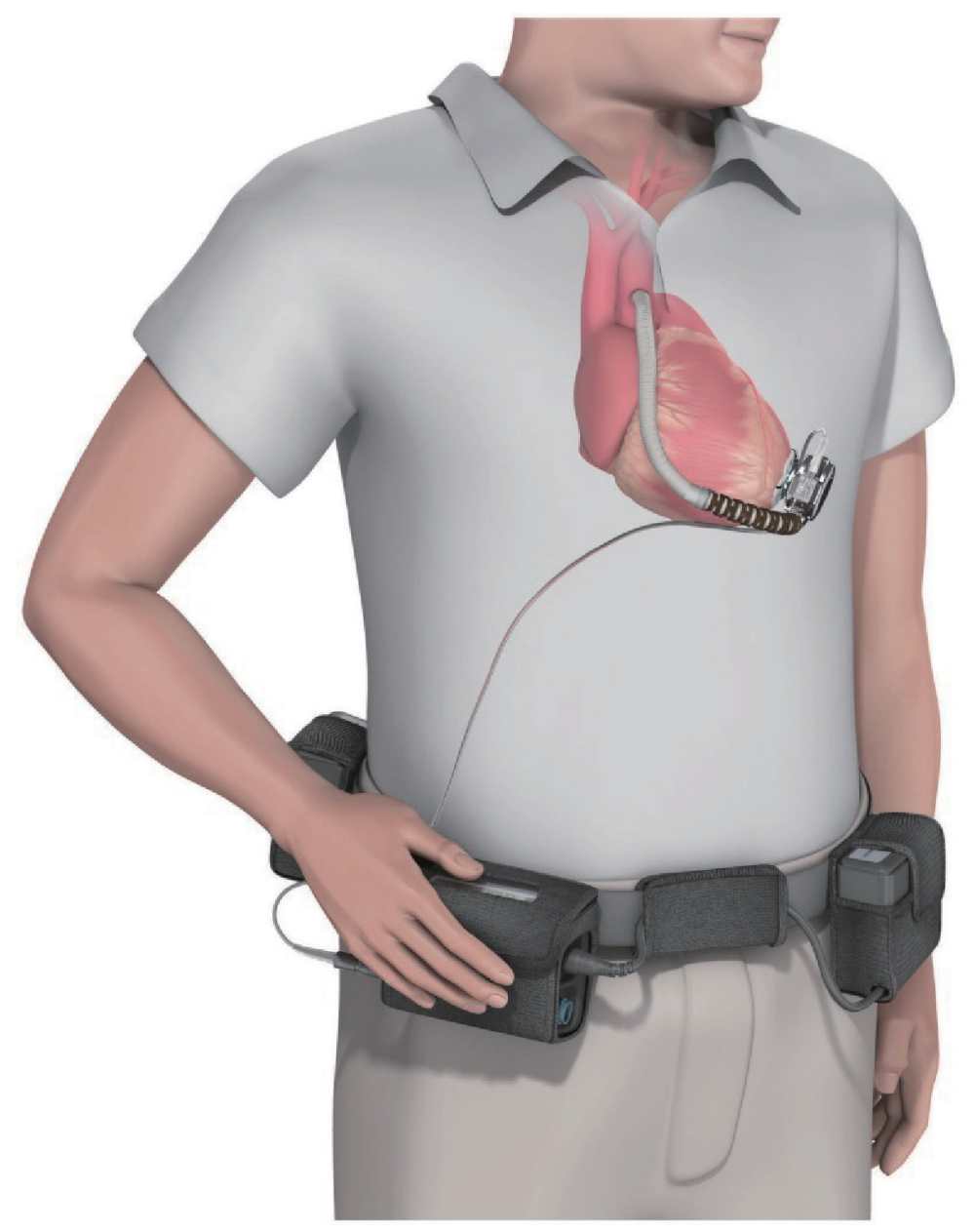

Figure 4. The HVAD (HeartWare Corp.) ventricular assist device. Copyright ${ }^{\odot} 2015$ Bonacchi, Harmelin, Bugetti and Sani. Creative commons license CC BY 4.0 (Permission obtained from original authors).

support, the inflow cannula can be placed in the right ventricle and the outflow unit in the pulmonary artery. The outflow cannula can also be connected to a peripheral vessel such as the femoral artery as in some paracorporeal devices like the Tandem and CentriMag VAD. Percutaneously implanted extracorporeal VADs such as Impella 2.5 can work as a single unit. The pump unit retains within a pump system, which aids the propagation of blood out to the systemic circulation. In pulsatile pumps, the diaphragm, pusher plates, and the one-way valve system establishes a pulsatile flow pattern that mimics that of the native heart. Conversely, VADs with continuous flow pumps have hydrodynamic or magnetically driven rotors within their pump unit that effectively propels blood with lesser noise [15-27]. They are also more portable and durable. Centrifugal flow VADs are preferred for longterm circulatory support because they have a lesser propensity to cause hemolysis as compared to VADs with axial flow pumps. Centrifugal pump VADs also have lesser coagulation promoting sites due to their intrinsic design. This is because centrifugal flow patterned VADs revolve at a lower revolutions per minute (RPM). A power module can be used when the external battery pack is exhausted with no access to spare batteries. These modules use AC and should be connected to an $\mathrm{AC}$ inlet through an AC power adapter [15-27].

\section{Healthcare innovations in VADs}

Current healthcare innovations in these devices include the development of a muscle powered VAD that can eliminate the need for a tethered driveline system by utilizing energy generated by muscle tissues like that of the latissimus dorsi muscle [15-23]. This may be made possible by the use of a muscle energy converter that works in unison with a controllable pacemaker-like stimulator which can synchronize the energy harnessed with the cardiac cycle and adequately power these devices at an optimum level. If properly actualized, this can help eliminate driveline-related infections and colonic erosions. Other areas of development include the generation of a non-blood contacting copulsation and counterpulsation VAD that can function by increasing systolic pulse pressure and cardiac output; decreasing afterload and increasing ventricular filling during diastole [15-24]. With this system in place, the major challenge of pump thrombosis can be resolved. Regard- 
ing this, the current HeartMate 3 VAD has been reported to have a wider flow conduit and an intrinsic pulsatile mechanism to decrease blood stasis and shear stress [24-27].

\section{Inflammatory response to VAD implantation}

Research by Radley et al has shown that certain cytokines, chemokines, and other biological molecules such as C-reactive protein are released in patients with heart failure, especially in those with underlying myocardial ischemia. These substances may include interleukin (IL)-1, tumor necrosis factor (TNF)alpha, IL-6, IL-8, IL-18, macrophage inflammatory protein 1 alpha, monocyte chemoattractant protein 1 , and galectin-3 [28]. Galectin-3 is upregulated in patients with heart failure and is often associated with fibrosis and ventricular remodeling. Other molecular entities released include cardiotrophin-1 which may induce myocardial hypertrophy. Alarmins like IL-33 and brain-natriuretic peptide are released in response to myocardial stress. IL-33 acts by binding to soluble suppression of tumorigenicity 2 (ST2) receptor. During myocardial remodeling secondary to ischemia injury, leukocyte count may be increased. When VADs are placed or implanted and cardiac function is re-established as the case may be, reverse remodeling of the myocardium takes place with a corresponding decrease in expression of galectin-3, ST2 receptor, brain natriuretic peptide and certain inflammatory cytokines such as TNF-alpha. On the other hand, cytokines like IL-6 and IL-8 may increase after implantation at varying levels. Reports by Ankersmit et al and Kimball et al [29, 30] have shown that after the implantation of a VAD, T-cell response tends to decrease in patients using some of these devices. This happens through the upregulation of cluster of differentiation (CD)95 (Fas receptor) which leads to apoptosis of CD4 and CD8 Tcells resulting in lymphopenia. Consequently, this increases the risk for opportunistic infections in such patients. The type of biomaterial used in the device affects the nature of inflammatory response. Cell-derived microparticles have also shown to be elevated secondary to shear stress [28-32]. Elevation of these biological molecules is indicative of vascular inflammation as some of these particles are derived from endothelial cells $[31,32]$.

\section{Materials and Methods}

All published articles screened for inclusion were obtained from the following databases: MEDLINE (PubMed), Cochrane, EB$\mathrm{SCO}$, clinicaltrials.gov, and international clinical trials registry. The search criteria were limited to only randomized control trials and observational studies published between 2010 and 2020. The type of intervention used for inclusion was restricted to VADs with continuous flow pumps (axial or centrifugal). Only original research articles were used. Grey literatures were also reviewed for inclusion. Articles were evaluated for inclusion by two independent reviewers. The Preferred Reporting Items for Systematic Reviews and Meta-Analyses (PRISMA) flow diagram was used to show study inclusion and exclu- sion at different levels. Research report was formulated using PRISMA guidelines. Studies with gastrointestinal bleeding (GIB) as a complication or adverse effect were screened for inclusion. Only articles with adult participants were included. The overall study sample accrued was divided into two groups which consisted of participants who received centrifugal flow VADs and participants who received axial flow devices as highlighted in all studies included. Only articles with English text were used. Both single and multicenter studies were evaluated and all studies included had a follow-up period of at least 6 months. After final evaluation of full-text articles and grey literatures, two randomized control trials and four observational studies were included; 85 studies were excluded as illustrated in the PRISMA flow diagram (Fig. 5). Data extraction was carried out in a tabular form and preliminary appraisal was done using Cochrane Collaboration's tool (RoB.2 with visualization through robviz) and Newcastle-Ottawa Scale (Fig. 6 and Table 1 [33-36]). Articles that failed to meet the stated inclusion criteria, containing non-English literature, possessing insufficient data or having a sample size of below 50 participants were excluded. Out of the six studies included, five were used for meta-analysis and all six were used for narrative synthesis. Search terms used included "continuous-flow ventricular assist devices", "continuous flow ventricular assist devices and their complications", "adverse effects in continuous-flow ventricular assist devices", "bleeding in ventricular assist devices", and "bleeding in continuous flow ventricular assist devices", "comparative study of outcomes in continuous-flow ventricular assist devices", "outcomes in continuous-flow ventricular assist devices", "gastrointestinal bleeding in continuous flow ventricular devices", and "gastrointestinal bleeding in axial and centrifugal-flow ventricular assist devices". Herein, we have also included a summary of all studies analyzed for both metaanalysis and narrative synthesis (Tables 2 - 7).

\section{Statistical analysis}

Data accrued from studies included in the meta-analysis were analyzed with " $\mathrm{R}$ " data analysis tool (version 4.0.0; release date: April 24th, 2020). The effect size used was odds ratio (OR) while the statistical model employed was the DerSimonian and Laird-Random-effects model (inverse variance method). Heterogeneity was assessed with the following functions: Tausquared (between-study variance), Cochran's Q (observed variation) and I-squared statistic (degree of heterogeneity). Other statistical measures applied include Fisher's exact test, confidence interval (CI) and $\mathrm{P}$ value. The crude OR of individual studied was confirmed with the "epiR" package within " $\mathrm{R}$ " data analysis tool. Pooled OR (overall effect size) was obtained during the meta-analysis. No subgroup analysis or meta-regression was done because the I-squared value was minimal. Data were presented in a pie chart, tabular forms, and forest plot. Publication bias was assessed using a funnel plot with standard error of the effect sizes (study precision) on the y-axis and the logs of the effect sizes ( $\log \mathrm{OR}$ ) on the x-axis. However, due to the paucity of articles on this subject, its reliability in detecting or excluding publication bias in this circumstance is questionable as the number of studies used in this research is limited. In ad- 


\section{PRSIMA FLOW DIAGRAM}
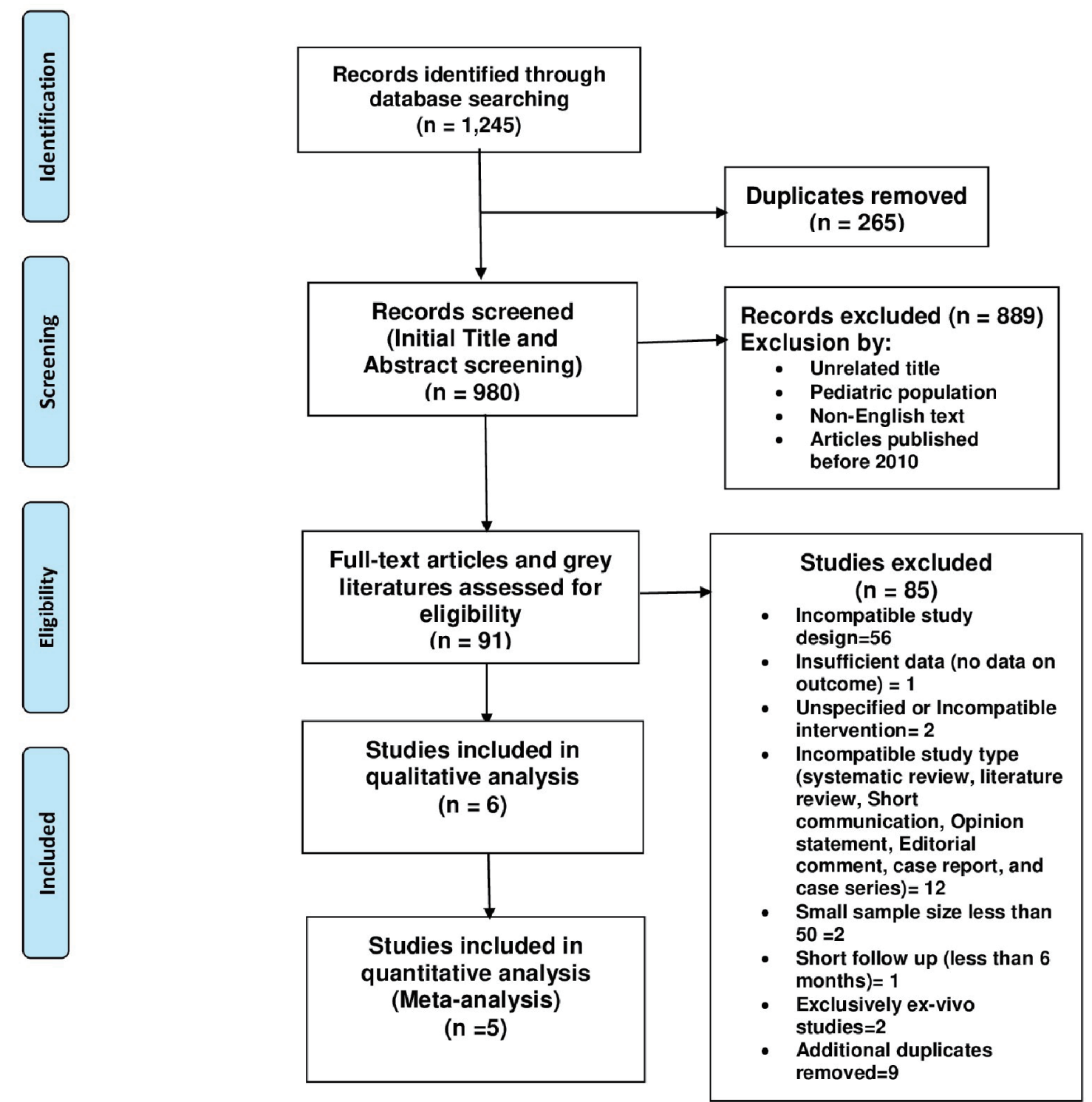

Figure 5. PRISMA flow diagram.

dition to the funnel plot, we utilized Egger's regression test as a secondary measure to assess the presence of publication bias. A trim and fill analysis was done to check for missing studies. Adjunct narrative synthesis was carried out in conjunction with scientific analysis and data presentation.

\section{Inclusion criteria}

Inclusion criteria are: 1) Adult participants; 2) Study design must specify device type; 3) Device type (intervention) accepted: centrifugal and axial flow VADs; 4) Parallel study design with two study arms (comparative); 5) Two study arms: centrifugal flow and axial flow group; 6) Methodology section must specify the number of participants in each study arm; 7) Intervention indications: bridge to transplant, bridge to recovery, and destination therapy; 8) Randomized control trials and observational studies only; 9) Sample size of 50 or greater; 10) Original article; 11) Published within the last 10 years (2010 2020); 12) Study outlined baseline characteristics of study participants; 13) GIB must be one of the outcomes; 14) The study outcome (GIB) must be reported separately for each study arm (centrifugal versus axial) for inclusion in the meta-analysis; 15) Single and multicenter studies (both accepted); 16) At least 6 months (180 days) follow-up after implantation; 17) No geographical restriction. 


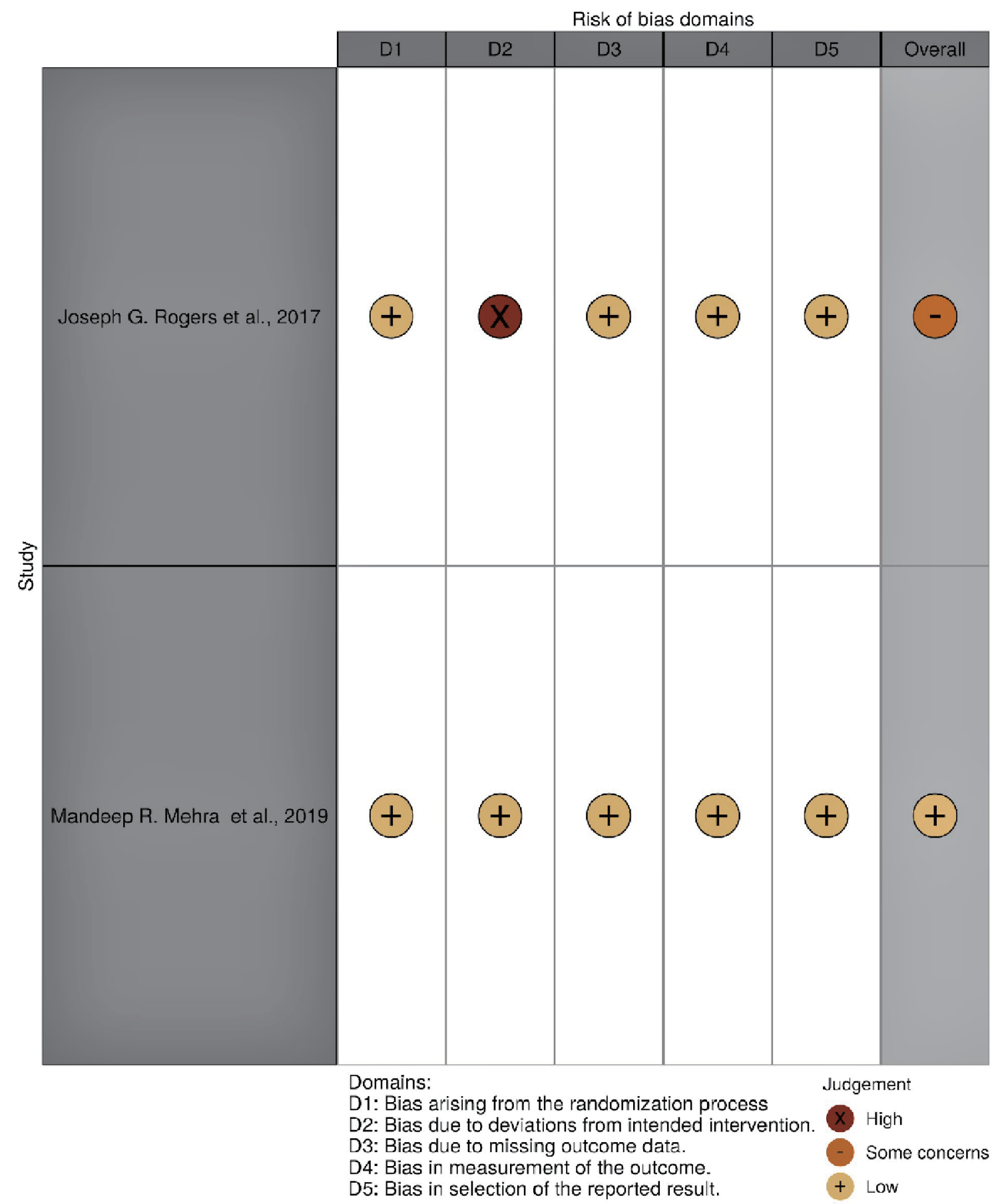

Bias arising from the randomization process Bias due to deviations from intended interventions Bias due to missing outcome data Bias in measurement of the outcome Bias in selection of the reported result Overall risk of bias

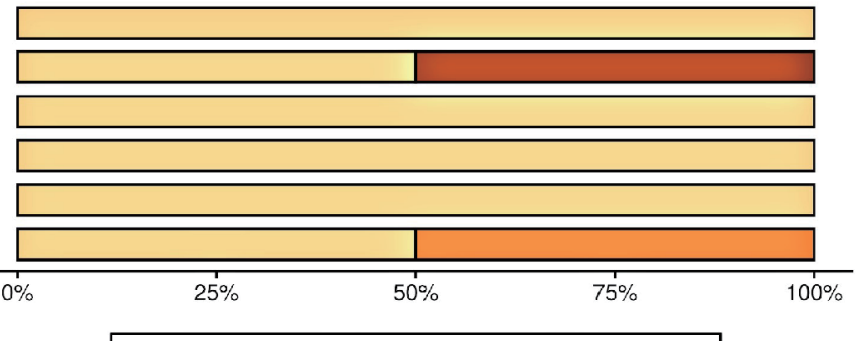

Figure 6. Outcome of RoB.2 quality assessment for all randomized control trials included (visualized with robviz). 
Table 1. Outcome of Newcastle-Ottawa Scale (NOS) Quality Assessment for All Observational Studies Included

\begin{tabular}{lllll}
\hline Author & $\begin{array}{l}\text { Selection } \\
(\text { max: 4 stars) }\end{array}$ & $\begin{array}{l}\text { Comparability } \\
\text { (max: 2 stars) }\end{array}$ & $\begin{array}{l}\text { Outcome/exposure } \\
\text { (max: 3 stars) }\end{array}$ & $\begin{array}{l}\text { NOS scale } \\
\text { (max: 9 stars) }\end{array}$ \\
\hline Gaffey et al, 2018 [33] & $* * *$ & $* *$ & $* * *$ & 8 \\
Sheikh et, 2015 [34] & $* *$ & $* *$ & $*$ & 5 \\
Stulak et al, 2016 [36] & $* * *$ & $*$ & $* *$ & 7 \\
Petrovic et al, 2016 [35] & $* * *$ & $* *$ & $7 *$ & 7 \\
\hline
\end{tabular}

\section{Exclusion criteria}

Exclusion criteria are: 1) Pediatric participants; 2) Sample size less than 50; 3) Non-English text; 4) Incompatible study design; 5) Insufficient data on outcome (GIB); 6) Articles published before 2010; 7) Incompatible study type; 8) Duplicates; 9) Pulsatile devices (incompatible intervention); 10) Exclusively ex vivo or non-human studies.

\section{Summary of included studies}

Rogers et al, 2017 [37] (used for meta-analysis and narrative synthesis)

This is a randomized multicenter control trial (non-blinded), with the type of randomization of permuted block with central randomization scheme. The devices types used were HeartWare (centrifugal flow) and HeartMate II (axial flow). A total of 155 events were reported (104 in HeartWare and 51 in HeartMate II); 297 participants were included in centrifugal flow group, and 148 in axial flow group (Table 2).

Mehra et al, 2019 [38] (used for meta-analysis and narrative synthesis)

This is a randomized multicenter control trial (non-blinded), with type of randomization of permuted blocks and implementation through an electronic data-capture system (eClinicalOS, Merge Healthcare). Stratification was done according to the study center. A total of 282 events were reported (126 in HeartMate 3 and 156 in HeartMate II). The device type(s)used were HeartMate 3 (centrifugal flow) and HeartMate II (axial flow). There were 516 participants in centrifugal flow group, and 512 in axial flow group, respectively (Table 3 ).

Gaffey et al, 2018 [33] (used for meta-analysis and narrative synthesis)

This is a single-center, retrospective cohort study. Fourteen

Table 2. Baseline Characteristics and Outcome as Reported by the Investigators

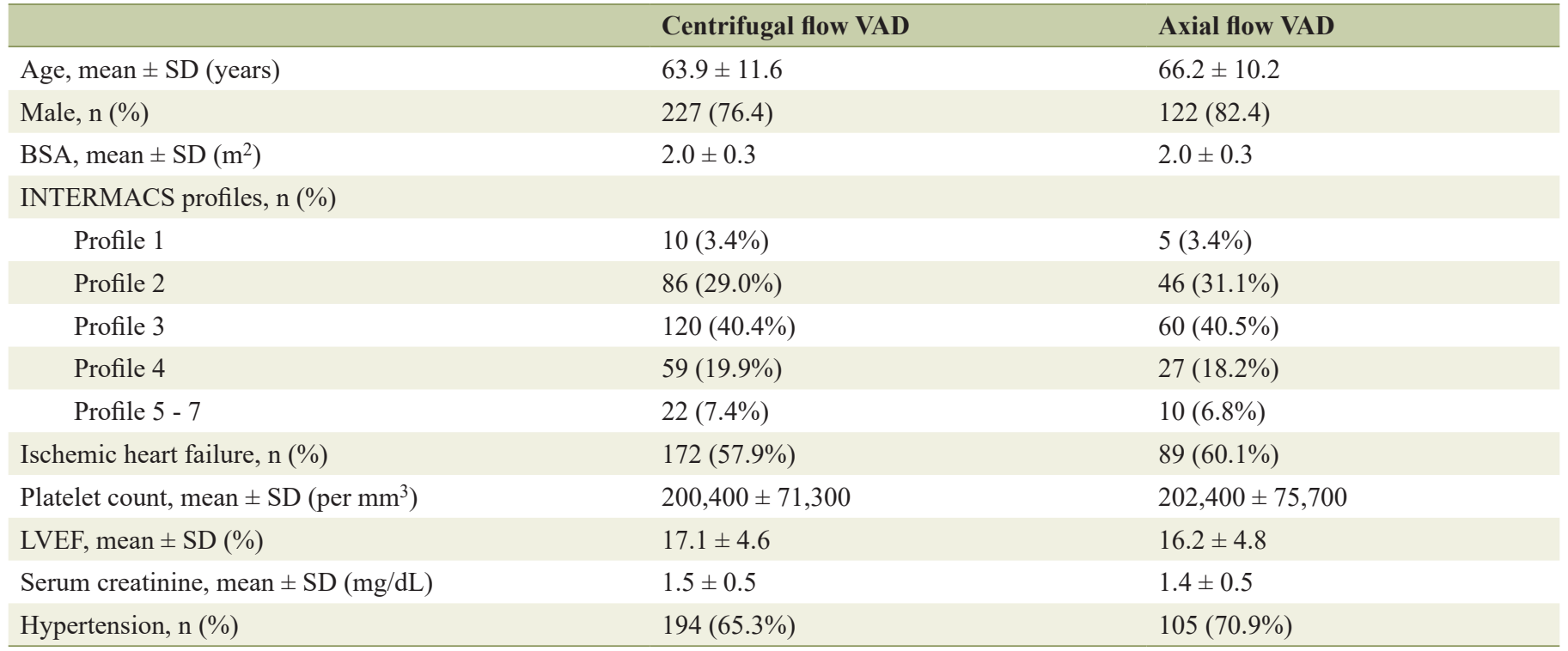

The target INR (IU) was 2.0 to 3.0 . Aspirin was given $81 \mathrm{mg}$ daily, then subsequently increased to $325 \mathrm{mg}$ daily. Follow-up duration is 24 months. There was no significant difference in GIB between both groups. INR: international normalized ratio; VAD: ventricular assist devices; BSA: body surface area; SD: standard deviation; LVEF: left ventricular ejection fraction; INTERMACS: Interagency Registry for Mechanically Assisted Circulatory Support; GIB: gastrointestinal bleeding. 
Table 3. Baseline Characteristics and Outcome as Reported by the Investigators

\begin{tabular}{lll}
\hline & Centrifugal flow VAD & Axial flow VAD \\
\hline Age, mean $\pm \mathrm{SD}($ years $)$ & $59 \pm 12$ & $60 \pm 12$ \\
BSA, mean $\pm \mathrm{SD}\left(\mathrm{m}^{2}\right)$ & $2.1 \pm 0.3$ & $2.1 \pm 0.3$ \\
Male, $\mathrm{n}(\%)$ & $411(79.7)$ & $419(81.8)$ \\
Ischemic heart disease, $\mathrm{n}(\%)$ & $216(41.9)$ & $240(46.9)$ \\
LVEF, mean \pm SD $(\%)$ & $17.3 \pm 5.1$ & $17.2 \pm 5.0$ \\
Mean arterial pressure, mean $\pm \mathrm{SD}(\mathrm{mm} \mathrm{Hg})$ & $79.2 \pm 10.4$ & $79.2 \pm 10.1$ \\
Cardiac index, mean $\pm \mathrm{SD}\left(\mathrm{L} / \mathrm{min} / \mathrm{m}^{2}\right)$ & $2.0 \pm 0.5$ & $2.0 \pm 0.6$ \\
Serum creatinine, mean $\pm \mathrm{SD}(\mathrm{mg} / \mathrm{dL})$ & $1.4 \pm 0.4$ & $1.4 \pm 0.4$ \\
INTERMACS profile, $\mathrm{n}(\%)$ & & $18(3.5)$ \\
$\quad$ Profile 1 & $11(2.1)$ & $146(28.5)$ \\
$\quad$ Profile 2 & $156(30.2)$ & $251(49.0)$ \\
$\quad$ Profile 3 & $272(52.7)$ & $82(16.0)$ \\
\hline Profile 4 & $67(13.0)$ & $15(2.9)$ \\
\hline
\end{tabular}

The target INR (IU) is 2.0 to $3.0 \mathrm{IU}$, with aspirin at $81 \mathrm{mg}$ to $325 \mathrm{mg}$ daily for each group. Follow-up duration is 24 months. Outcome: GIB was lower in centrifugal flow VAD group as compared to axial flow VAD group. It was reported that for every 10 patients who received centrifugal flow VAD instead of axial flow VAD, 3.6 GIB events were avoided in 2 years. Nevertheless, a significant residual risk of bleeding was reported, with the investigators prompting further investigations to see if a reduction in antiplatelet or anticoagulation therapy will be beneficial to these patients. INR: international normalized ratio; VAD: ventricular assist devices; BSA: body surface area; SD: standard deviation; LVEF: left ventricular ejection fraction; INTERMACS: Interagency Registry for Mechanically Assisted Circulatory Support; GIB: gastrointestinal bleeding.

Table 4. Baseline Characteristics and Outcome as Reported by the Investigators

\begin{tabular}{|c|c|c|}
\hline & Centrifugal flow VAD & Axial flow VAD \\
\hline Age (years) & $57.2+14.6$ & $56.5+13.9$ \\
\hline Male, n (\%) & $27(81.8)$ & $83(79.1)$ \\
\hline Platelet $\left(\times 10^{3} / \mathrm{mm}^{3}\right)$ & $191.9+83.4$ & $184.9+69.8$ \\
\hline Prothrombin time (s) & $52.3+3.9$ & $52.6+2.5$ \\
\hline \multicolumn{3}{|l|}{ Past medical history, n (\%) } \\
\hline Gastrointestinal bleeding & $1(2.9)$ & $4(3.9)$ \\
\hline Hypertension & $19(55.9)$ & $55(52.9)$ \\
\hline Chronic renal insufficiency & $10(30.3)$ & $37(35.2)$ \\
\hline Profile 2 & $14(41.2)$ & $39(37.1)$ \\
\hline Profile 3 & $15(44.1)$ & $36(34.3)$ \\
\hline Profile 4 & - & $14(13.3)$ \\
\hline Profile 5 & - & $1(0.9)$ \\
\hline
\end{tabular}

The target INR (IU) is 2.0 to $3.0 \mathrm{IU} ; 325 \mathrm{mg}$ of aspirin. Follow-up: 36 months. Outcome: there was no significant difference in GIB between both groups. INR: international normalized ratio; VAD: ventricular assist devices; SD: standard deviation; LVEF: left ventricular ejection fraction; INTERMACS: Interagency Registry for Mechanically Assisted Circulatory Support; GIB: gastrointestinal bleeding; BMI: body mass index. 
Table 5. Baseline Characteristics and Outcome as Reported by the Investigators

\begin{tabular}{lll} 
& Centrifugal flow VAD & Axial flow VAD \\
\hline Age, mean \pm SD (years) & $58 \pm 11$ & $59 \pm 11$ \\
Men (\%) & $61 \%$ & $88 \%$ \\
Median time to first GIB (months) & $3.5 \pm 0.98$ & $8.2 \pm 3.5$ \\
Experienced more than one GIB & $10 \%$ & $9 \%$ \\
LVEF (\%) & - & - \\
Mean follow-up duration (years) & 1.3 & 1.0 \\
\hline
\end{tabular}

Target INR (IU): as reported by the investigators, the mean INR (IU) at the time of GIB did not differ between the two groups: $2.57 \pm 2.3$ for HeartMate II and $2.56 \pm 1.9$ for HeartWare VAD, P value $=0.9$. Outcome: There was no significant difference in the cumulative incidence of GIB between both groups. INR: international normalized ratio; VAD: ventricular assist devices; SD: standard deviation; LVEF: left ventricular ejection fraction; GIB: gastrointestinal bleeding.

index events were reported (two in HeartWare group and 12 in HeartMate II group). Device types used were HeartWare (centrifugal flow) and HeartMate II (axial flow). Thirty-four participants were included in the centrifugal flow group, and 105 in axial flow group, respectively (Table 4).
Sheikh et al, 2015 [34] (used for both meta-analysis and narrative synthesis)

This is a single-center, retrospective cohort study. Thirty-six

Table 6. Baseline Characteristics and Outcome as Reported by the Investigators

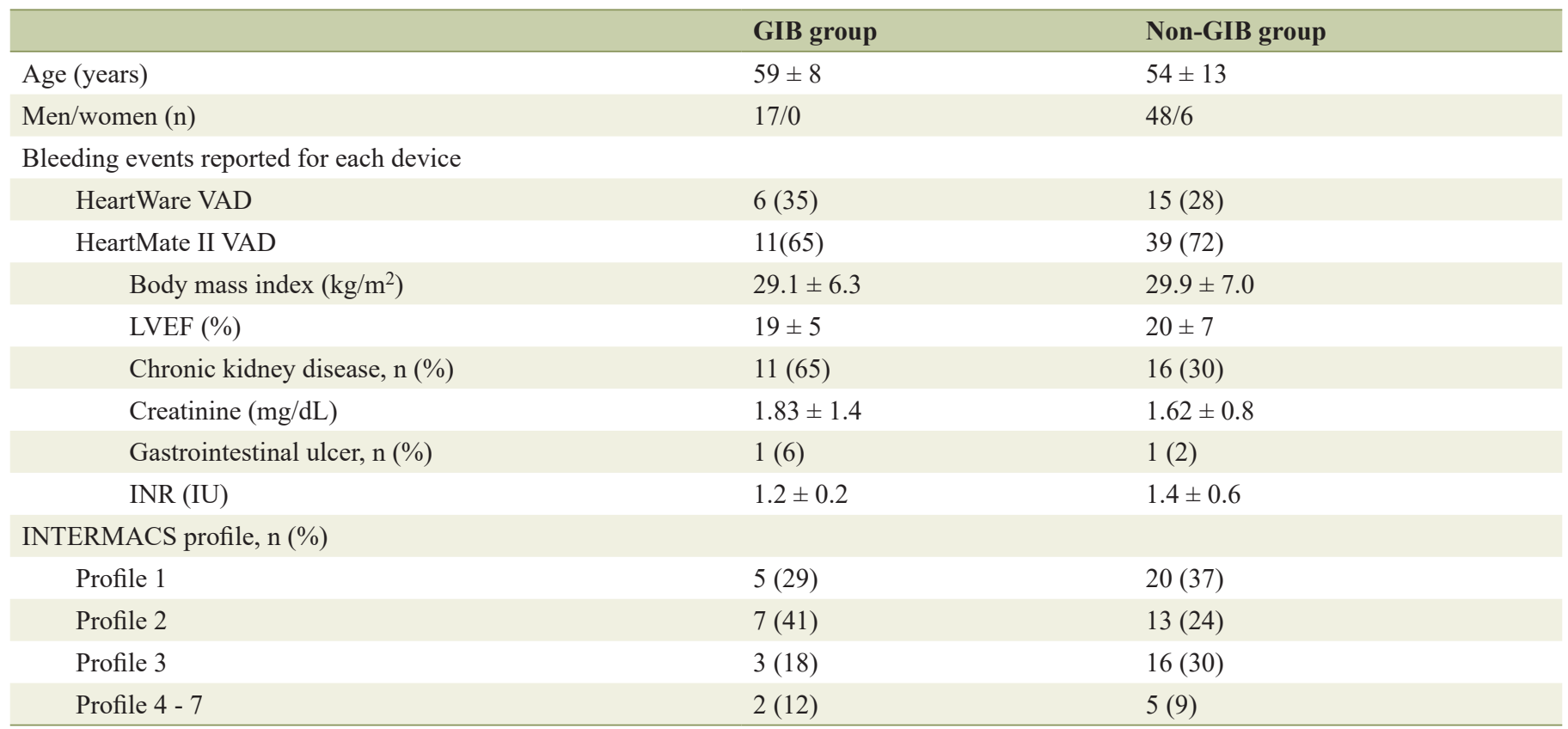

Results are expressed as mean \pm SD. Median follow-up time: 6 months; maximum: 23 months. Anticoagulation regimen: 81 mg aspirin and a target INR (IU) of 2.0 - 3.0. At the first GIB event, target INR range was reduced to $1.5-2.0$ IU and supplemented by 81 mg aspirin. A second event prompted the cessation of antiplatelet agents and continued use of warfarin at INR level of $1.5-2.0 \mathrm{IU}$. Outcome: As reported by the investigators, patients with GIB were all men with 17 episodes of GIB reported (index cases). The mean age of patients in the GIB group was $59 \pm 8$ years; with 11 out of 17 episodes occurring within the first month after the pump implantation. Fourteen patients had a single episode, and three patients had multiple bleeding episodes. All patients with multiple GIBs had a HeartMate II pump and the only risk factor significant for GIB was the presence of chronic kidney disease; with $\mathrm{OR}=3.95 ; 95 \% \mathrm{Cl}: 1.21-12.84 ; \mathrm{P}=0.02$. All patients with $\mathrm{GIB}$ presented with anemia, eight with melena, one with hematemesis, six without an obvious bleeding source, and two with only positive guaiac stool tests. Esophagogastroduodenoscopy (EGD), small-bowel enteroscopy, and colonoscopy confirmed bleeding sources in 13 patients. Arteriovenous malformations (AVMs) were identified as the major source of bleeding in eight out of 13 patients (61\%). In two patients endoscopy confirmed bleeding from two sources during the same GIB episode. One patient had both gastric and jejunal AVM. The other patient had rectal AVM and sigmoid colon diverticulosis. In one patient, the small bowel AVM was identified only by on-table push enteroscopy because EGD, colonoscopy, nuclear medicine study, and angiograms failed to diagnose the bleeding source. Only one patient with multiple GIB episodes was diagnosed with coagulopathy, and one patient had GIB whenever his INR value was above 2 IU. GIB was noted to occur more often within the first month after implantation. The presence of blood type $O$ was not associated with increased risk of bleeding. INR: international normalized ratio; VAD: ventricular assist devices; SD: standard deviation; LVEF: left ventricular ejection fraction; INTERMACS: Interagency Registry for Mechanically Assisted Circulatory Support; GIB: gastrointestinal bleeding; OR: odds ratio; Cl: confidence interval. 
Table 7. Baseline Characteristics and Outcome as Reported by Investigators

\begin{tabular}{lll} 
& Centrifugal flow VAD & Axial flow VAD \\
\hline Age (years), median (range) & $58.3(18.0-82.0)$ & $59.5(18.0-82.0)$ \\
BMI $\left(\mathrm{kg} / \mathrm{m}^{2}\right)$, median (range) & $27.6(2.0-142.2)$ & $28.5(16.3-43.7)$ \\
Male, $\mathrm{n}(\%)$ & $126(72)$ & $465(83)$ \\
Female, $\mathrm{n}(\%)$ & $48(28)$ & $95(17)$ \\
Creatinine (mg/dL), mean (SD) & $1.3(0.5)$ & $1.4(0.5)$ \\
Preoperative inotropes, n (\%) & $126(74.6)$ & $425(76.4)$ \\
INTERMACS profiles, $\mathrm{n}(\%)$ & & $74(13)$ \\
$\quad$ Profile 1 & $19(11)$ & $125(22)$ \\
$\quad$ Profile 2 & $25(14)$ & $185(33)$ \\
$\quad$ Profile 3 & $78(45)$ & $176(32)$ \\
Profile 4 - 7 & $52(30)$ & $2.8(1.1)$ \\
\hline Mean (SD) & $2.9(1.1)$ & \\
\hline
\end{tabular}

Follow-up: median of 1 year (max: 10 years). Anticoagulation regimen: all patients in the HeartWare (centrifugal flow VAD) group were maintained on $325 \mathrm{mg}$ of aspirin. Dipyridamole was used almost routinely by one of the institutions and selectively by others. Clopidogrel (Plavix) was utilized on a case by case basis when pump thrombus or hemolysis occurred. Patients were routinely bridged with unfractionated intravenous heparin when INR fell below 2.0 IU. INR range for the HeartMate II (axial flow VAD) group was 1.5 - 2.5 and 2.0 - 3.0 for the HeartWare group. Additional outcomes reported: the investigators reported older age at implant, preoperative inotrope use and preoperative creatinine were reported as independent predictors of gastrointestinal bleeding. The risk of bleeding in the HeartMate II group was found to be highest when the INR was > 2.5 IU. INR: international normalized ratio; VAD: ventricular assist devices; SD: standard deviation; LVEF: left ventricular ejection fraction; INTERMACS: Interagency Registry for Mechanically Assisted Circulatory Support; GIB: gastrointestinal bleeding; AVM: arteriovenous malformation; BMI: body mass index.

events were reported, $19(18 \%)$ in HeartWare and 17 in HeartMate II (26\%). Device types used were HeartWare (centrifugal flow) and HeartMate II (axial flow). There were 104 participants in centrifugal flow group, and 66 in axial flow group (Table 5).

Petrovic et al, 2016 [35] (used for both meta-analysis and narrative synthesis)

This is a single-center, retrospective (with case (GIB group) and control (non-GIB group) groups) study. Device types used were HeartWare (centrifugal flow) and HeartMate II (axial flow). Seventeen index cases/events were reported (six in HeartWare and 11 in HeartMate II); with HeartWare: events/ group sample size $(\mathrm{e} / \mathrm{n})=6 / 21$; and HeartMate II: events/group sample size $(e / n)=11 / 50$. Twenty-one participants were included in the centrifugal flow group, and 50 in axial flow group (Table 6).

\section{Stulak et al, 2016 [36] (used for only narrative synthesis)}

This is a multicenter retrospective cohort study. Device types used are HeartWare (centrifugal flow) and HeartMate II (axial flow). There were 174 participants in centrifugal flow group, and 560 in axial flow group, respectively. Events for both groups were not stated (reason for inclusion in only narrative synthesis), but the investigators reported that there was no significant difference in time-related cumulative risk of GIB between both groups $(\mathrm{P}=0.18)$. Both devices were not independently associated with GIB $(\mathrm{P}=0.63)$ (Table 7).

Table 8. Sample Size (N) Across All Studies and Percentage of Participants in Each Study

\begin{tabular}{lll} 
Included studies & Sample size (n) & Percentage distribution \\
\hline Rogers et al, 2017 [37] & 446 & $17.23 \%$ \\
Mehra et al, 2019 [38] & 1,028 & $39.72 \%$ \\
Gaffey et al, 2018 [33] & 139 & $5.37 \%$ \\
Sheikh et al, 2015 [34] & 170 & $6.57 \%$ \\
Petrovic et al, 2016 [35] & 71 & $2.74 \%$ \\
Stulak et al, 2016 [36] & 734 & $28.36 \%$ \\
Total & 2,588 & $100.00 \%$ \\
\hline
\end{tabular}

The initial study size was 2,588, but with the study of Stulak et al [36] excluded from the meta-analysis, the meta-analysis consisted of 1,854 participants. The study of Stulak et al [36] was used concomitantly with other studies for narrative synthesis. 


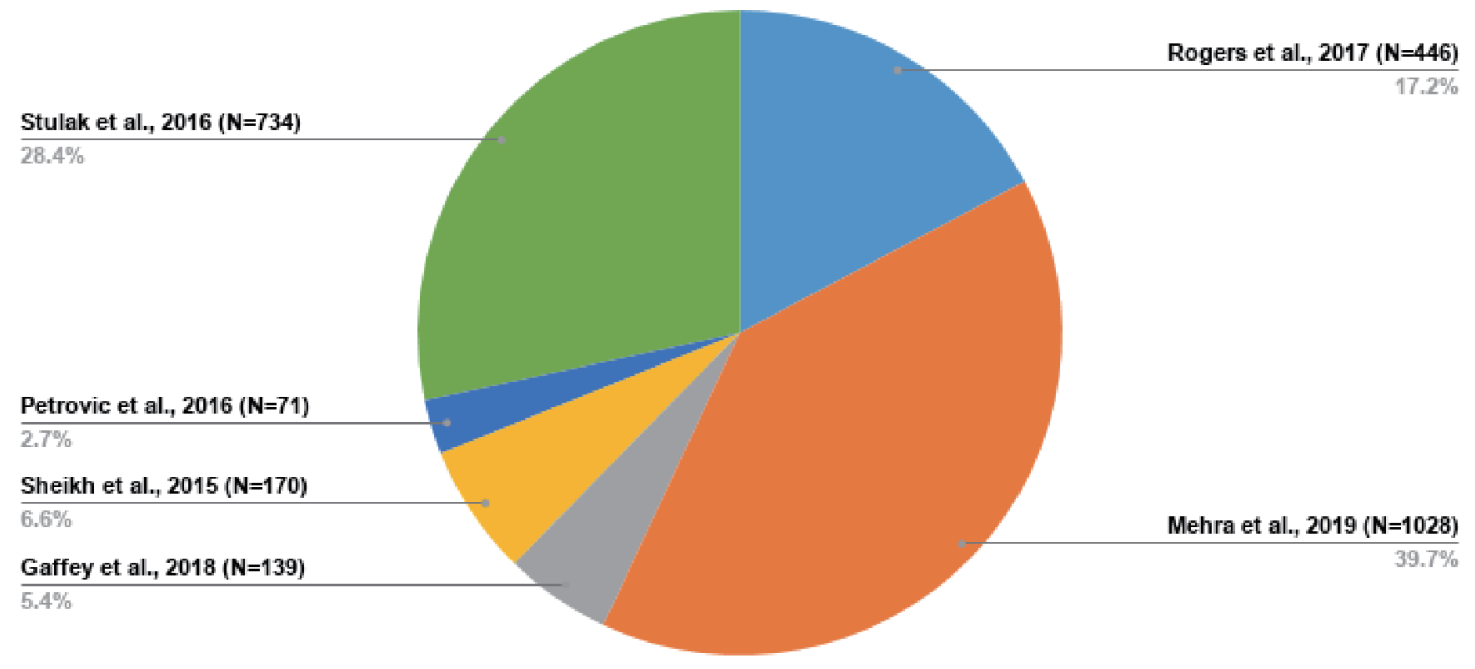

Figure 7. Sample distribution across all studies included.

The initial study size was 2,588 , but with the study of Stulak et al [36] excluded from the meta-analysis, the meta-analysis consisted of 1,854 participants as shown in Table 8 [33-38]. Figure 7 further illustrates the sample distribution. The study of Stulak et al [36] was used concomitantly with other studies for narrative synthesis.

The Review Board Approval (IRB) approval was not required because we did not use any confidential patient information in this study. All studies used for this research are publicly accessible as well. And the ethical compliance with human was not applicable because no human subjects were used in this research.

\section{Results}

Through this meta-analysis, we determined that the occurrence of GIB was not significantly different between both groups; with OR: 0.81; 95\% CI: 0.65 - 1.00; P value $=0.05$ (Fig. 8). Out of all the studies included in the meta-analysis, the study of Mehra et al [38] showed a significant difference in GIB between both groups with more events occurring in the axial flow

\section{Author(s) and Year}

Odds Ratio $[95 \% \mathrm{Cl}]$

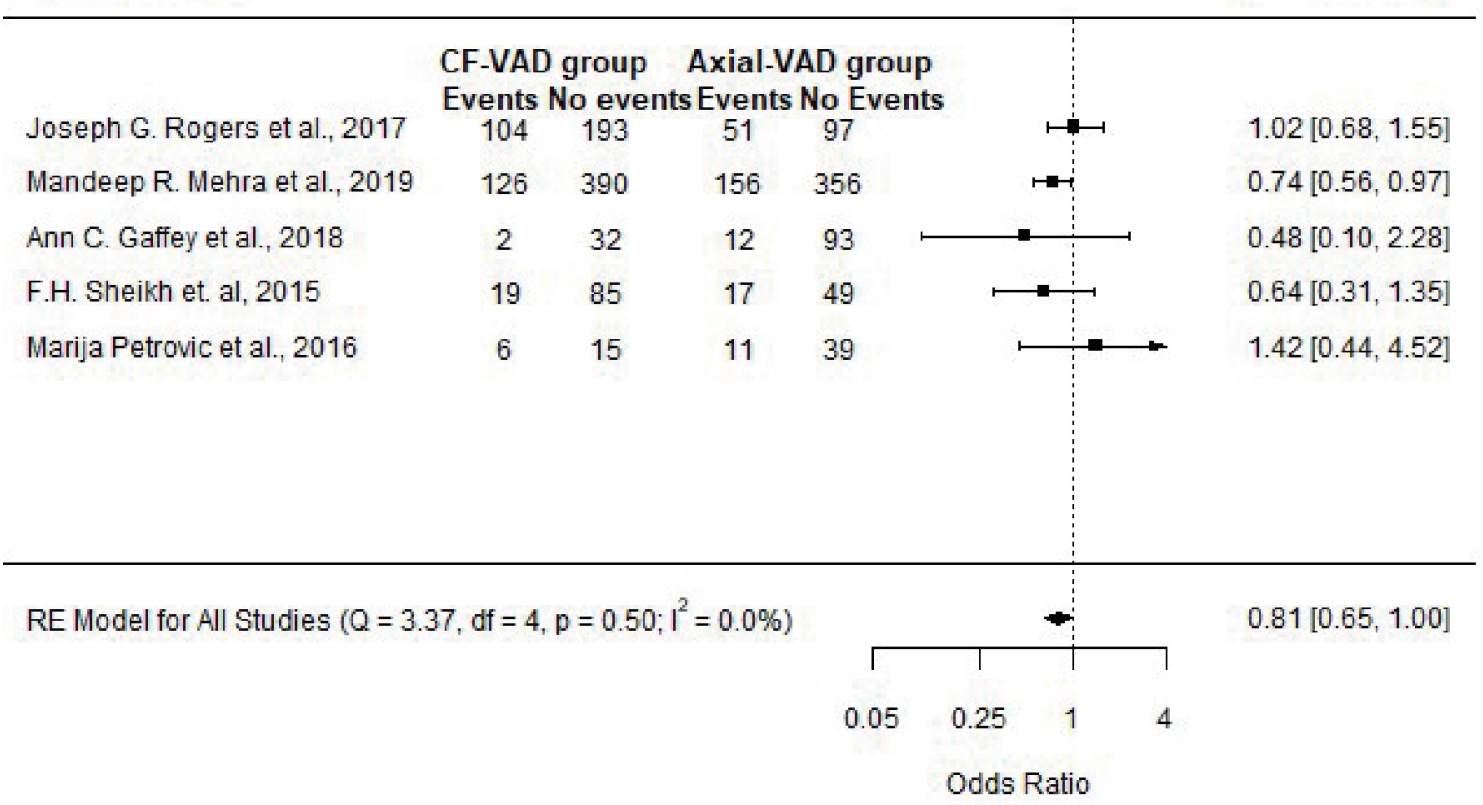

Figure 8. Forest plot showing the effect sizes for all studies included and the pooled effect size (pooled odds ratio) at a confidence level of $95 \%$. 
Table 9. Number of Participants With Events in Each Group per Study

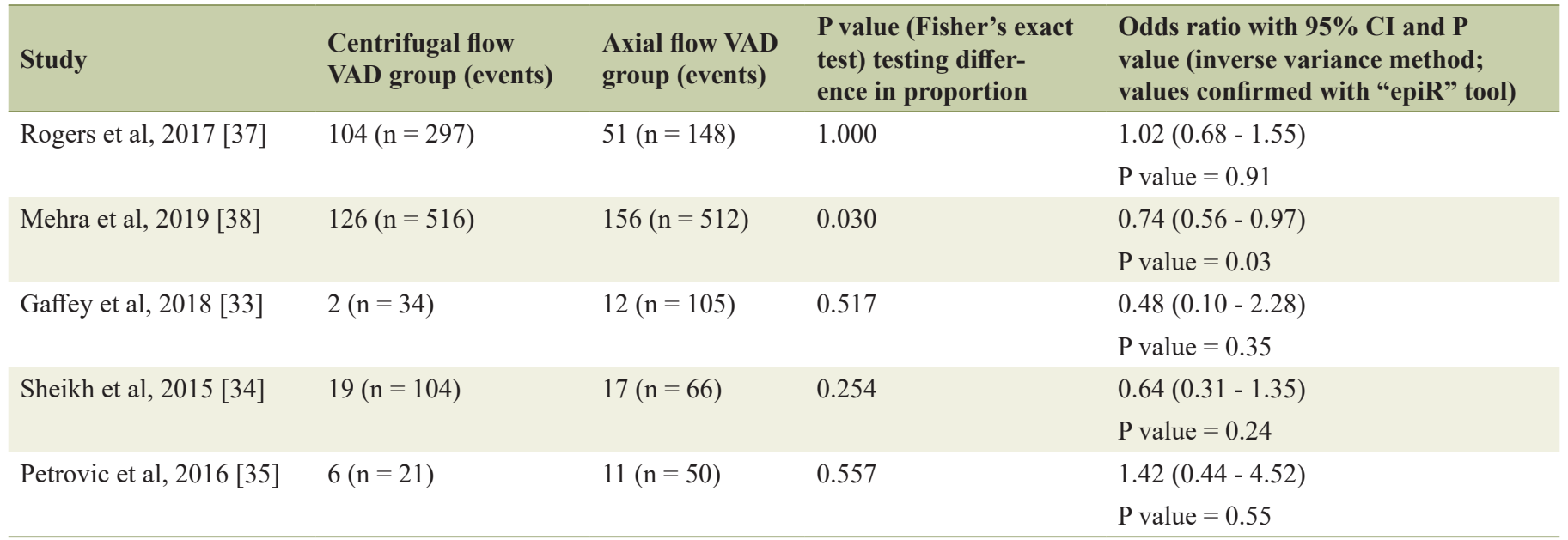

VAD: ventricular assist device; $\mathrm{n}$ : sample size within each group per study; Cl: confidence interval (95\% limit).

VAD group as compared to the centrifugal flow VAD group; with OR: $0.74 ; 95 \%$ CI: $0.56-0.97 ; \mathrm{P}$ value $=0.03$ (Table 9 $[33-35,37,38])$. Upon completion of the meta-analysis, the between-study variance (Tau-squared) was zero (0), $\mathrm{SE}=0.06$. The observed variation (Cochran's $\mathrm{Q}=3.37 ; \mathrm{P}=0.50$ ) in the estimated effect sizes was lower than the expected variation $(\mathrm{df}=$ 4); thereby producing a negative measure of excess dispersion; thus the I-squared value is $0 \%$ (minimal). Egger's regression test was not statistically significant, $\mathrm{P}=0.93$. However, we feel the small sample size of this research reduced the statistical power of the tests used in this meta-analysis; such as the test for heterogeneity, measure of overall effect and the Egger's regression test. All points on the funnel plot were distributed within the $95 \%$ CI region with no asymmetry observed (Fig.
9). Trim and fill analysis showed no missing studies on the left; $\mathrm{SE}=1.68$. The prediction interval of the true effect is the same as that of the pooled estimate derived from the meta-analysis because I-squared is $0 \%$.

\section{Discussion}

According to the INTERMACS (Interagency Registry for Mechanically Assisted Circulatory Support) definition, GIB is defined as any clinically suspected or documented bleeding from the gastrointestinal tract indicated by a newly identified drop in hemoglobin level and the appearance of melena, hematochezia, hematemesis, or guaiac-positive stool [33-38]. In

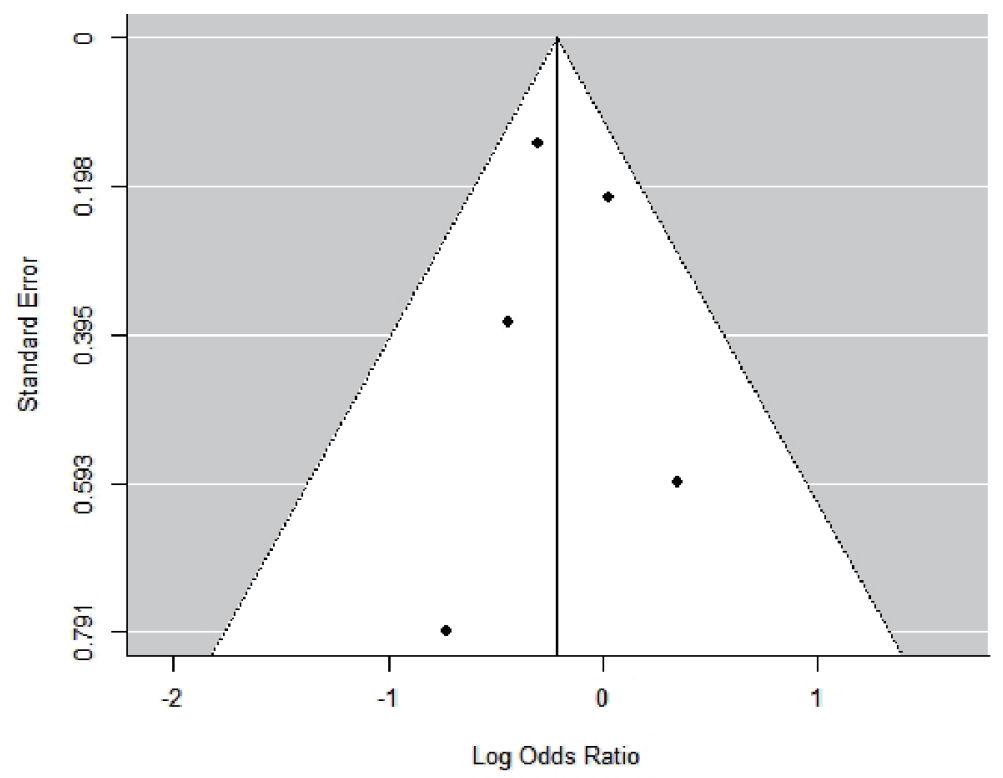

Figure 9. Funnel plot showing the distribution of effect estimates against their standard error (index of precision). The effect estimates are distributed as log odds ratio. 
entirety, bleeding is one of the most common adverse effects reported in these patients. GIB has been stated to be the most common cause of 30-day readmission and tends to affect the quality of life and survival outcome of patients using these devices. Petrovic et al [35] stated that out of the 17 GIB events that were observed, 11 occurred within the first 30 days after device implantation. GIB events can be classified based on their site. Upper GIB occurs proximal to the ligament of Treitz while lower GIB occurs distal to the ligament of Treitz. Common gastrointestinal lesions implicated in GIB from the studies analyzed include Mallory-Weiss tear, gastric AVM, gastric ulcer, gastric polyp, duodenal AVM, jejunal AVM, cecal AVM, cecal ulcer, distal transverse colon ulcer, sigmoid colon diverticulosis, sigmoid colon AVM, and rectal AVM [33-38]. Inciting factors of GIB in these patients are known to be multifactorial. Some predisposing factors identified include acquired von Willebrand disease, anticoagulation or antiplatelet therapy and other independent risk factors such as older age, preoperative inotrope use, underlying coagulopathy, previous history of GIB, chronic kidney disease and thrombocytopenia [33-38]. Blood group $\mathrm{O}$ was not reported to be associated with an increased risk of bleeding even though they are known to have lower von Willebrand factor (VWF) at baseline when compared to other blood groups [33-38]. Bleeding due to acquired von Willebrand disease is said to occur through the breakdown of high molecular weight VWFs by ADAMST-13 with a relative increase in VWF fragments secondary to the conformational change induced by the high shear stress emanating from these devices [33-36, 39-46]. This process is synonymous to Heyde's syndrome which is known to occur in patients with severe aortic stenosis. AVMs or angiodysplasias are linked to the high flow shear stress that results in dilatation of the submucosal veins which eventually leads to their formation [33-36, 39-46]. In some cases, they have also been postulated to occur when the blood level of high molecular weight VWFs significantly decreases. The use of anticoagulants or antiplatelets is known to promulgate the risk of bleeding. This challenge has made it difficult for physicians to balance the risk of bleeding with thrombosis or thromboembolism, which are all common complications [47-49]. Preliminary management strategies utilized in the treatment of GIB in these patients include hemodynamic stabilization, initial discernment of abnormalities in coagulation profile, blood count and organ dysfunction (prothrombin time (PT)/international normalized ratio (INR), bleeding time, complete blood count, creatinine, and blood urea nitrogen), stoppage of anticoagulants and antiplatelets therapy; adjustment of pump speed and the utilization of octreotide and proton pump inhibitors [47-49]. Adjustment of pump speed should be done under echocardiographic guidance. Blood products are administered based on their corresponding laboratory metrics (hemoglobin, PT/INR, and platelet count). In refractory cases or GIB induced by warfarin; vitamin $\mathrm{K}$, prothrombin complex concentrate, cryoprecipitate, or fresh frozen plasma may be used to curtail bleeding [50-52]. Nonetheless, caution should be exercised to avoid pump thrombosis or thromboembolism. Common diagnostic procedures include esophagogastroduodenoscopy (EGD) or colonoscopy. In cases where the source of bleeding cannot be identified, the following measures have been reported to be used; capsule endoscopy, balloon enter- oscopy, tagged red cell scan and angiogram (mesenteric). Besides the preliminary management techniques outlined earlier, other therapeutic options employed in the management of GIB in these patients include electrocoagulation, laser photocoagulation, injection of hemostatic solutions, endoscopic ligation, use of argon plasma, clipping, and transcatheter embolization [53-55]. In severe cases with poor hemodynamics, surgical approach may be utilized as a diagnostic and therapeutic measure. Anticoagulation or antiplatelet therapy is often held until complete resolution of GIB. On the contrary, patients with high risk of thrombosis or thromboembolic events like those with mechanical heart valves and non-valvular atrial fibrillation, may require warfarin to be maintained at the lower limits of the optimal therapeutic INR range depending on the type of device used and the institutional guidelines. With regards to therapeutic INR range, Stulak et al [36] reported that the risk of bleeding in the axial flow VAD group (HeartMate II) was found to be highest when the INR was $>2.5 \mathrm{IU}$, and recommendations were later made to maintain the INR level at 1.5 to $2.5 \mathrm{IU}$. The INR range for the centrifugal flow VAD group (HeartWare) was placed at 2.0 to $3.0 \mathrm{IU}$ mainly due to the risk of pump thrombosis. The risk of pump thrombosis was noted to decrease further when warfarin was supplemented with an antiplatelet agent. In addition to bridging with heparin, the therapeutic INR level for all studies mostly ranged between 2.0 to $3.0 \mathrm{IU}$, with all upper limits at $\leq 3.0 \mathrm{IU}$. Adjustments were made as complications arose. The commonly used antiplatelet agent was aspirin $(81-325 \mathrm{mg})$. Other antiplatelet agents like dipyridamole and clopidogrel (Plavix) were added in some cases. The use of direct thrombin and factor Xa inhibitors was not mentioned in any of the studies. Adequate blood pressure control and post-operative monitoring is advised to attain better outcomes [47-55]. Few studies have explored the use of genotype-guided warfarin therapy in these patients with most reporting promising outcomes with its use [56-58].

\section{Limitations}

The main limitation of this meta-analysis is the sample size due to paucity of articles on the topic, with reference to the device in question. We implore that an extensive meta-analysis should be undertaken once more studies are made available. Another limitation we will like to bring to light is that the devices identified from the studies were limited to HeartMate II, HeartWare, and HeartMate 3. We are not sure if the outcome of this meta-analysis can be applied to other types of continuous flow VADs. Even though we included studies with at least 6 months duration of follow-up with the maximum duration of follow-up reported to be 36 months, we still feel the discordance in the follow-up duration between the studies may have affected the estimated outcome reported.

\section{Conclusions}

The result of this meta-analysis indicates that there is no significant difference in the occurrence of GIB between both groups 
(centrifugal and axial flow VAD). Regardless of the type of continuous flow VAD used, GIB continues to be a major challenge in these patients. We recommend that more research should be conducted on this topic to aid the feasibility of a broader metaanalysis. Data on outcomes should be explicitly outlined and possible confounding factors should be controlled. In addition to our initial recommendation, we propose that more studies should be carried out to explore the integration of precision medicine with warfarin dosing based on the genetic composition of the VKORC1 and CYP2C9 genotypes of individual patients. The role of cell-derived microparticles in association with these devices also requires further investigation.

\section{Acknowledgments}

None to declare.

\section{Financial Disclosure}

None to declare.

\section{Conflict of Interest}

None to declare.

\section{Informed Consent}

Not applicable.

\section{Author Contributions}

Dr. Asuka and Dr. Pak formulated the research design and carried out the initial manuscript preparation. Dr. Asuka carried out the data analysis. Dr. Thiess and Dr. Torres III appraised the study results and revised the manuscript.

\section{Data Availability}

All the data obtained from the meta-analysis are within the article. For further correspondence, you may reach out to the corresponding author.

\section{Abbreviations}

VAD: ventricular assist device; LVAD: left ventricular assist device; AVM: arteriovenous malformation; GIB: gastrointestinal bleeding; INTERMACS: Interagency Registry for $\mathrm{Me}-$ chanically Assisted Circulatory Support; NYHA: New York Heart Association; ECMO: extracorporeal membrane oxygenation; TAH: total artificial heart; IABP: intra-aortic balloon pump

\section{References}

1. Center for Disease Control and Prevention. Heart disease: heart failure. URI: https:/www.cdc.gov/heartdisease/ heart failure.html [Accessed: June 11th, 2020].

2. Emory Healthcare. Heart and vascular: conditions and treatments. URI: https://www.emoryhealthcare.org/heartvascular/wellness/heart-failure-statistics.html [Accessed: June 11th, 2020].

3. Montefiore. Transplantation: heart transplantation. Accessed: June 11th, 2020. URI: https://www.montefiore. org/heart-transplantation.

4. Severino P, Mather PJ, Pucci M, D'Amato A, Mariani MV, Infusino F, Birtolo LI, et al. Advanced heart failure and end-stage heart failure: does a difference exist. Diagnostics (Basel). 2019;9(4):170.

5. Peura JL, Colvin-Adams M, Francis GS, Grady KL, Hoffman TM, Jessup M, John R, et al. Recommendations for the use of mechanical circulatory support: device strategies and patient selection: a scientific statement from the American Heart Association. Circulation. 2012;126(22):2648-2667.

6. St. Luke's Health system. Biventricular assist device implantation. Available at: https://www.saintlukeshealthsystem.org/health-library/biventricular-assist-device-implAntation [Accessed June 11th, 2020].

7. University of Utah Health. Types of ventricular assist devices; [Accessed June 11th, 2020]; URI: https://healthcare.utah.edu/transplant/cms/vad-types.php.

8. Khazanie P, Hammill BG, Patel CB, Eapen ZJ, Peterson ED, Rogers JG, Milano CA, et al. Trends in the use and outcomes of ventricular assist devices among medicare beneficiaries, 2006 through 2011. J Am Coll Cardiol. 2014;63(14):1395-1404.

9. Mancini D, Colombo PC. Left ventricular assist devices: a rapidly evolving alternative to transplant. J Am Coll Cardiol. 2015;65(23):2542-2555.

10. Starling RC, Estep JD, Horstmanshof DA, Milano CA, Stehlik J, Shah KB, Bruckner BA, et al. Risk assessment and comparative effectiveness of left ventricular assist device and medical management in ambulatory heart failure patients: the ROADMAP study 2-year results. JACC Heart Fail. 2017;5(7):518-527.

11. Kirklin JK, Naftel DC, Pagani FD, Kormos RL, Stevenson L, Miller M, Young JB. Long-term mechanical circulatory support (destination therapy): on track to compete with heart transplantation? J Thorac Cardiovasc Surg. 2012;144(3):584-603; discussion 597-588.

12. Guidelines Pocket Cards: Mechanical circulatory support. American Heart Association. Available at: https:// professional.heart.org/professional/GuidelinesStaments/ GuidelinesPocketcards/UCM_475319_Guidelines-Pocketcards.jsp [Accessed: June 11̄th, 2020].

13. Samson R, Jaiswal A, Smart F, LeJemtel TH. Incidence of pump thrombosis in heartmate II during destination therapy. The VAD Journal. 2016;2:Article 16.

14. Li S, Beckman JA, Welch NG, Bjelkengren J, Masri SC, Minami E, Stempien-Otero A, et al. Accuracy of Dop- 
pler blood pressure measurement in continuous-flow left ventricular assist device patients. ESC Heart Fail. 2019;6(4):793-798.

15. Han J, Trumble DR. Cardiac assist devices: early concepts, current technologies, and future innovations. Bioengineering (Basel). 2019;6(1).

16. Texas Heart Institute. Ventricular Assist Devices. [Accessed: June 11th, 2020]; URI: http://www.texasheart. org/HIC/Topics/Proced/vads.cfm.

17. Northwestern Medicine. Ventricular Assist Devices. [Accessed: June 11th, 2020]; URI: https://www.nm.org/ conditions-and-care-areas/treatments/ventricular-assistdevices.

18. Sherwin J, Thompson E, Hill KD, Watt K, Lodge AJ, Gonzalez D, Hornik CP. Clinical pharmacology considerations for children supported with ventricular assist devices. Cardiol Young. 2018;28(9):1082-1090.

19. Adachi I, Jaquiss RD. Mechanical circulatory support in children. Curr Cardiol Rev. 2016;12(2):132-140.

20. Di Nardo M, MacLaren G, Marano M, Cecchetti C, Bernaschi P, Amodeo A. ECLS in Pediatric Cardiac Patients. Front Pediatr. 2016;4:109.

21. Imamura $T$, Kinugawa K. Indication of ventricular assist device therapy in patients with INTERMACS profile 4-7. Ann Thorac Cardiovasc Surg. 2016;22(5):271-274.

22. Sabashnikov A, Mohite PN, Weymann A, Patil NP, Hedger M, Saez DG, Zych B, et al. Outcomes after implantation of 139 full-support continuous-flow left ventricular assist devices as a bridge to transplantation. Eur J Cardiothorac Surg. 2014;46(5):e59-66.

23. Jaquiss RD, Humpl T, Canter CE, Morales DL, Rosenthal DN, Fraser CD, Jr. Postapproval Outcomes: The Berlin Heart EXCOR Pediatric in North America. ASAIO J. 2017;63(2):193-197.

24. Bourque K, Cotter C, Dague C, Harjes D, Dur O, Duhamel J, Spink K, et al. Design rationale and preclinical evaluation of the heartmate 3 left ventricular assist system for hemocompatibility. ASAIO J. 2016;62(4):375-383.

25. U.S. Food and Drug Administration. Recently approved devices: 2019 Device Approvals. [Accessed: June 11th, 2020]. URI: https://www.fda.gov/medical-devices/recently-approved-devices/2019-device-approvals.

26. Potapov EV, Antonides C, Crespo-Leiro MG, Combes A, Farber G, Hannan MM, Kukucka M, et al. 2019 EACTS expert consensus on long-term mechanical circulatory support. Eur J Cardiothorac Surg. 2019;56(2):230-270.

27. Abott. Momentum 3 Trial: Publications and presentations from the Momentum 3 trial. [Accessed: June 11th, 2020]. URI: https://www.cardiovascular.abbott/us/en/momentum3publications.html.

28. Radley G, Pieper IL, Ali S, Bhatti F, Thornton CA. The inflammatory response to ventricular assist devices. Front Immunol. 2018;9:2651.

29. Ankersmit HJ, Tugulea S, Spanier T, Weinberg AD, Artrip JH, Burke EM, Flannery M, et al. Activationinduced T-cell death and immune dysfunction after implantation of left-ventricular assist device. Lancet. 1999;354(9178):550-555.

30. Kimball PM, Flattery M, McDougan F, Kasirajan V.
Cellular immunity impaired among patients on left ventricular assist device for 6 months. Ann Thorac Surg. 2008;85(5):1656-1661.

31. Nascimbene A, Hernandez R, George JK, Parker A, Bergeron AL, Pradhan S, Vijayan KV, et al. Association between cell-derived microparticles and adverse events in patients with nonpulsatile left ventricular assist devices. J Heart Lung Transplant. 2014;33(5):470-477.

32. Jeske WP, Walenga JM, Menapace B, Schwartz J, Bakhos M. Blood cell microparticles as biomarkers of hemostatic abnormalities in patients with implanted cardiac assist devices. Biomark Med. 2016;10(10):1095-1104.

33. Gaffey AC, Chen CW, Chung JJ, Han J, Bermudez CA, Wald J, Atluri P. Is there a difference in bleeding after left ventricular assist device implant: centrifugal versus axial? J Cardiothorac Surg. 2018;13(1):22.

34. Sheikh FH, Majure DT, Salcedo J, Hofmeyer M, Ruiz G, Rodrigo ME, Elliott T, et al. Comparison of GI Bleeding Rates between Axial Flow and Centrifugal Flow LVADs. J Heart Lung Transplant. 2015;34(4):231-232.

35. Petrovic M, Nathan S, Radovancevic R, Rajapreyar I, Dasher KJ, Akay MH, Akkanti B. Adverse events in continuous-flow LVAD recipients: gastrointestinal bleeding is still notable? The VAD Journal. 2016;2. Article 23.

36. Stulak JM, Davis ME, Haglund N, Dunlay S, Cowger J, Shah P, Pagani FD, et al. Adverse events in contemporary continuous-flow left ventricular assist devices: A multiinstitutional comparison shows significant differences. J Thorac Cardiovasc Surg. 2016;151(1):177-189.

37. Rogers JG, Pagani FD, Tatooles AJ, Bhat G, Slaughter MS, Birks EJ, Boyce SW, et al. Intrapericardial left ventricular assist device for advanced heart failure. N Engl J Med. 2017;376(5):451-460.

38. Mehra MR, Uriel N, Naka Y, Cleveland JC, Jr., Yuzefpolskaya M, Salerno CT, Walsh MN, et al. A fully magnetically levitated left ventricular assist device - final report. N Engl J Med. 2019;380(17):1618-1627.

39. Cushing K, Kushnir V. Gastrointestinal bleeding following LVAD Placement from top to bottom. Dig Dis Sci. 2016;61(6):1440-1447.

40. Gurvits GE, Fradkov E. Bleeding with the artificial heart: Gastrointestinal hemorrhage in CF-LVAD patients. World J Gastroenterol. 2017;23(22):3945-3953.

41. Geetha Bhat, Mukesh Gopalakrishnan, Ashim Aggarwal. Gastrointestinal bleeding with continuous flow left ventricular assist devices. In: Komamura K. (Ed.). Recent advances in the field of ventricular assist devices. 2013:5560.

42. Parikh NS, Cool J, Karas MG, Boehme AK, Kamel H. Stroke risk and mortality in patients with ventricular assist devices. Stroke. 2016;47(11):2702-2706.

43. Mehra MR, Stewart GC, Uber PA. The vexing problem of thrombosis in long-term mechanical circulatory support. J Heart Lung Transplant. 2014;33(1):1-11.

44. Joy PS, Kumar G, Guddati AK, Bhama JK, Cadaret LM. Risk factors and outcomes of gastrointestinal bleeding in left ventricular assist device recipients. Am J Cardiol. 2016;117(2):240-244.

45. Starling RC, Moazami N, Silvestry SC, Ewald G, Rog- 
ers JG, Milano CA, Rame JE, et al. Unexpected abrupt increase in left ventricular assist device thrombosis. N Engl J Med. 2014;370(1):33-40.

46. Thomas SS, Nahumi N, Han J, Lippel M, Colombo P, Yuzefpolskaya M, Takayama H, et al. Pre-operative mortality risk assessment in patients with continuous-flow left ventricular assist devices: application of the HeartMate II risk score. J Heart Lung Transplant. 2014;33(7):675-681.

47. Adatya S, Bennett MK. Anticoagulation management in mechanical circulatory support. J Thorac Dis. 2015;7(12):2129-2138.

48. Donna Mancini. Practical management of long-term mechanical circulatory support devices. UpToDate. [Accessed: June 11th, 2020]; URI: https://www.uptodate. com/contents/practical-management-of-long-term-mechanical-circulatory-support-devices.

49. Slaughter MS, Pagani FD, Rogers JG, Miller LW, Sun B, Russell SD, Starling RC, et al. Clinical management of continuous-flow left ventricular assist devices in advanced heart failure. J Heart Lung Transplant. 2010;29(4 Suppl):S1-39.

50. Jennings DL, Rimsans J, Connors JM. Prothrombin complex concentrate for warfarin reversal in patients with continuous-flow left ventricular assist devices: a narrative review. ASAIO J. 2020;66(5):482-488.

51. Maguire M, Fuh L, Goldstein JN, Marshall AL, Levine M, Howell ML, Parry BA, et al. Thromboembolic risk of 4-factor prothrombin complex concentrate versus fresh frozen plasma for urgent warfarin reversal in the emergency department. West J Emerg Med. 2019;20(4):619625.

52. Kuroski JE, Young S. Comparison of the safety and effi- cacy between 3 -factor and 4-factor prothrombin complex concentrates for the reversal of warfarin. Am J Emerg Med. 2017;35(6):871-874.

53. Cotts WG, McGee EC, Jr., Myers SL, Naftel DC, Young JB, Kirklin JK, Grady KL. Predictors of hospital length of stay after implantation of a left ventricular assist device: an analysis of the INTERMACS registry. J Heart Lung Transplant. 2014;33(7):682-688.

54. Cowger J, Sundareswaran K, Rogers JG, Park SJ, Pagani FD, Bhat G, Jaski B, et al. Predicting survival in patients receiving continuous flow left ventricular assist devices: the HeartMate II risk score. J Am Coll Cardiol. 2013;61(3):313-321.

55. Ahsan I, Faraz A, Mehmood A, Ullah W, Ghani AR. Clinical approach to manage gastrointestinal bleeding with a Left Ventricular Assist Device (LVAD). Cureus. 2019;11(12):e6341.

56. Topkara VK, Knotts RJ, Jennings DL, Garan AR, Levin AP, Breskin A, Castagna F, et al. Effect of CYP2C9 and $\mathrm{VKORC1}$ gene variants on warfarin response in patients with continuous-flow left ventricular assist devices. ASAIO J. 2016;62(5):558-564.

57. Nakagita K, Wada K, Mukai Y, Uno T, Nishino R, Matsuda $\mathrm{S}$, Takenaka $\mathrm{H}$, et al. Effects of vitamin $\mathrm{K}$ epoxide reductase complex 1 gene polymorphisms on warfarin control in Japanese patients with left ventricular assist devices (LVAD). Eur J Clin Pharmacol. 2018;74(7):885894.

58. Awad M, Czer LS, Soliman C, Mirocha J, Ruzza A, Pinzas J, Rihbany K, et al. Prevalence of warfarin genotype polymorphisms in patients with mechanical circulatory support. ASAIO J. 2015;61(4):391-396. 